OPEN ACCESS

Edited by:

Bing $X u$,

Xiamen University, China

Reviewed by:

Carlo V. Bruschi,

University of Salzburg, Austria Valentina Tosato,

AREA Science Park, Italy

*Correspondence:

Huan-You Wang

huw003@health.ucsd.edu

Specialty section:

This article was submitted to

Molecular and Cellular Oncology,

a section of the journal

Frontiers in Oncology

Received: 22 October 2020 Accepted: 15 February 2021

Published: 10 March 2021

Citation: Wang HY, Sokol ES, Goodman AM, Feldman AL and Mulroney CM (2021)

Case Report: Multiple Chromosomal

Translocations Including Novel CIITA-CREBBP Fusion and Mutations

in a Follicular Lymphoma.

Front. Oncol. 11:620435

doi: 10.3389/fonc.2021.620435

\section{Case Report: Multiple Chromosomal Translocations Including Novel CIITA-CREBBP Fusion and Mutations in a Follicular Lymphoma}

\author{
Huan-You Wang ${ }^{1 *}$, Ethan S. Sokol ${ }^{2}$, Aaron M. Goodman ${ }^{3}$, Andrew L. Feldman ${ }^{4}$ and \\ Carolyn M. Mulroney ${ }^{3}$
}

'Division of Laboratory and Genomic Medicine, Department of Pathology, University of California San Diego Health System, La Jolla, CA, United States, ${ }^{2}$ Foundation Medicine, Cambridge, MA, United States, ${ }^{3}$ Division of Blood and Bone Marrow Transplant, Department of Medicine, University of California San Diego Health System, La Jolla, CA, United States,

${ }^{4}$ Department of Laboratory Medicine and Pathology, Mayo Clinic, Rochester, MN, United States

The pathogenesis of follicular lymphoma is a multi-step process, in which chromosomal translocation between immunoglobulin heavy chain $(\mathrm{lgH})$ and anti-apoptotic B-cell lymphoma 2 (BCL2), namely $\mathrm{lgH}-\mathrm{BCL} 2$, is an earliest step, followed by other genetic/genomic alterations including but not limited to mutation of CREB binding protein (CREBBP). MHC class II transactivator (CIITA) is a transcription regulator responsible for expression of MHC class II molecules including HLA-DR in human. We report herein a novel fusion gene involving CIITA and CREBBP in a patient with a low-grade follicular lymphoma (FL) but with high Ki-67 proliferation index. In addition, our patient also harbors CREBBP mutation. Together, we postulate that total loss of CREBBP function may contribute, in part, to the lymphoma genesis. Furthermore, this patient has addition rare (TBL1XR1-TP63) and common (IgH-BCL2) chromosomal translocations and multiple mutations including BCL2, BRAF, MUTYH, and STAT6.

Keywords: CIITA, CIITA-CREBBP, follicular lymphoma, BCL 2, CREBBP, TBL1XR1-TP63

\section{INTRODUCTION}

Non-random, balanced, and less commonly unbalanced translocations involving immunoglobulin heavy chain (IgH) and less frequently light chains kappa (IgK) and lambda (IgL) with another oncogene / transcription factor are common in B-cell lymphomas, and are the contributing factor in cancer transformation as well-demonstrated in S cerevisiae (1,2); however, translocations involving two oncogenes / transcription factors are rare in lymphoma in comparison to acute myeloid leukemias (AML) in either human (3) or yeast (4). Examples of balanced translocations in Bcell lymphomas include $\mathrm{t}(14 ; 18)$ involving IgH-BCL2 in follicular lymphoma (FL) $(5)$ and $\mathrm{t}(8 ; 14)$ involving MYC-IgH in Burkitt lymphoma (6). The major histocompatibility complex (MHC) class II transactivator (CIITA) is a master transcriptional regulator of diverse genes expressions beyond MHC class II and class I (7). CIITA is constitutively expressed in B-cells (8), and knock-down of CIITA or mutated CIITA in B-cells results in the absence of MHC-DR expression in the B-cell line L23 from swine (9). CREBBP, which binds to cAMP-response element-binding protein (CREB), also known as CBP short for CREB Binding Protein, is a lysine acetyltransferase functioning as one of the components of master coactivator along with p300. Although CIITA fusion with BX648577 
in classical Hodgkin lymphoma (cHL) and primary mediastinal large B-cell lymphoma (PMBL) (10), and CREBBP fusions with other genes in AML (11) have been reported, CIITA fusion with CREBBP, namely CIITA-CREBBP, has not yet been reported in the English literature to the best of our knowledge. Herein we report a novel CIITA-CREBBP fusion in a histological low-grade FL but with high Ki-67 proliferation index. In addition to CIITACREBBP, the case reported here has several additional interesting molecular and clinical features. First, in contrast to CIITABX648577 fusions detected in CHL and PMBL where CIITA lost its function with resultant lack of HLA-DR expression among the lymphoma cells, to the contrary, the CIITA in CIITA-CREBBP from our case is predicted to retain its function but with predicted loss of function of CREBBP; secondly, this case harbors other two balanced translocations, namely the commonly encountered signature $t(14 ; 18)$ involving IgH-BCL2 and a very rarely reported TBL1XR1-TP63; thirdly, this case exhibits multiple mutations including BCL2, BRAF, CREBBP, MUTYH, and STAT6; finally this lymphoma is a low-grade FL based on histology but shows high Ki-67 proliferation index and early clinical relapse.

\section{CLINICAL SUMMARY}

A 41-year old woman presented with extensive and bulky abdominal lymphadenopathy with small bowel involvement by large B-cell lymphoma (DLBCL) $(\sim 20-30 \%)$ arising in association with grade 3b FL ( 70-80\%) in early February 2018. She was treated on a clinical trial with R-CHOP [rituximab, cyclophosphamide, hydroxydaunomycin, oncovin (vincristine), and prednisone] and Pembrolizumab for six cycles, which was started right after the diagnosis and was completed by June 2018. Her first post treatment PET/CT scan was concerning for residual abdominal focus of disease, however, it was subsequently resolved without further intervention and she achieved a complete metabolic response by PET/CT in September of 2018. In November 2018 she developed back pain resembling prior symptoms of lymphoma and eventually underwent additional evaluations including two additional biopsies: an excisional biopsy from mesenteric lymph nodes in early March of 2019, which showed grade $1 \mathrm{FL}$ with high Ki-67 (see below), and a core biopsy from abdomen in early June 2019, again showing grade $1 \mathrm{FL}$ with high Ki-67 (not shown). A bone marrow biopsy at the same time with the early June 2019 abdominal tissue core biopsy showed no lymphomatous involvement. She was further treated with three cycles of R-ICE (rituximan, ifosfamide, carboplatin, and etoposide) from 6/29/2019 to 8/9/2019. Shortly after receiving BEAM conditioning in September 2019, she underwent autologous hematopoietic stem cell transplant, and she remains remission as of today. The patient had not received radiation therapy at all.

\section{PATHOLOGIC AND MOLECULAR FINDINGS}

H\&E of the excised mesenteric lymph node from early March 2019 showed total effacement of architecture by similarly sized

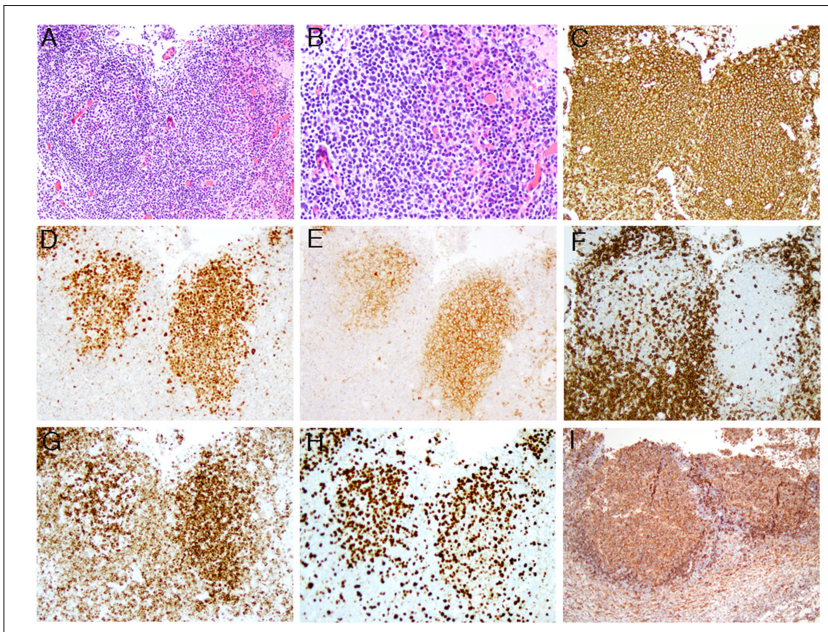

FIGURE 1 | The composite microphotographs of low-grade FL with high proliferation index. (A,B) The follicular lymphoma cells are mainly centrocytes with $<5$ centroblasts per high power field [the original magnifications of $(\mathbf{A}, \mathbf{B})$ are 100x and 200x, respectively]; (C-E) the lymphoma cells are positive for CD20 (C), BCL6 (D), and CD10 (E) [the original magnifications of (C-E) are 100x, 100x, and 100x, respectively]; (F-H) In comparison to negative CD3 (F) the lymphoma cells are positive for $\mathrm{BCL2}$ (G) with high $\mathrm{Ki}-67$ proliferation index (H) [the original magnifications for (F-H) are 100x, 100x, and 100x, respectively]; (I) The lymphoma cells are positive for HLA-DR (the original magnification is 100x).

and shaped neoplastic secondary follicles with $<5$ centroblasts per high power field, thus consistent with a grade $1 \mathrm{FL}$ (Figures 1A,B, the original magnifications of $\mathrm{A}$ and $\mathrm{B}$ are $100 \mathrm{X}$ and 200X, respectively). The neoplastic cells within the secondary follicles are positive for CD20 (1C), CD10 (1D), and BCL6 (1E) (the original magnifications of C-E are 100X, 100x, and $100 \mathrm{X}$, respectively). In comparison to negative CD3 (1F) (original magnification of 100X), the neoplastic cells show co-expression of BCL2 (1G) (original magnification of 100X), thus confirming the morphologic impression of FL; However, Ki-67 proliferation index was high $(>30 \%)$ (Figure 1H) (original magnification of $100 \mathrm{X}$ ), therefore a final diagnosis of histologically grade $1 \mathrm{FL}$ with high Ki-67 proliferation index was rendered. The neoplastic cells are also positive for HLA-DR (1I) (original magnification of 100X).

Karyotypic analysis by G-banding yielded no metaphase cells, and fluorescence in situ hybridization was not performed. Comprehensive genomic profiling employing next generation sequencing (NGS) using formalin-fixed paraffin-embedded (FFPE) tissue block from early March 2019 at both the RNA and DNA levels performed at the Foundation Medicine (Cambridge, MA, USA), which interrogated a panel of 671 genes, showed the following chromosomal translocations: CIITA-CREBBP (Figure 2), IGH-BCL2 (not shown), and TBL1XR1-TP63 (not shown), and genes mutations (not shown): BCL2 A60V, BRAF D594G, CREBBP R1446H, MUTYH Q400*, and STAT6 D419G. Her tumor mutational burden was 9 Muts/Mb, designated as intermediate. 


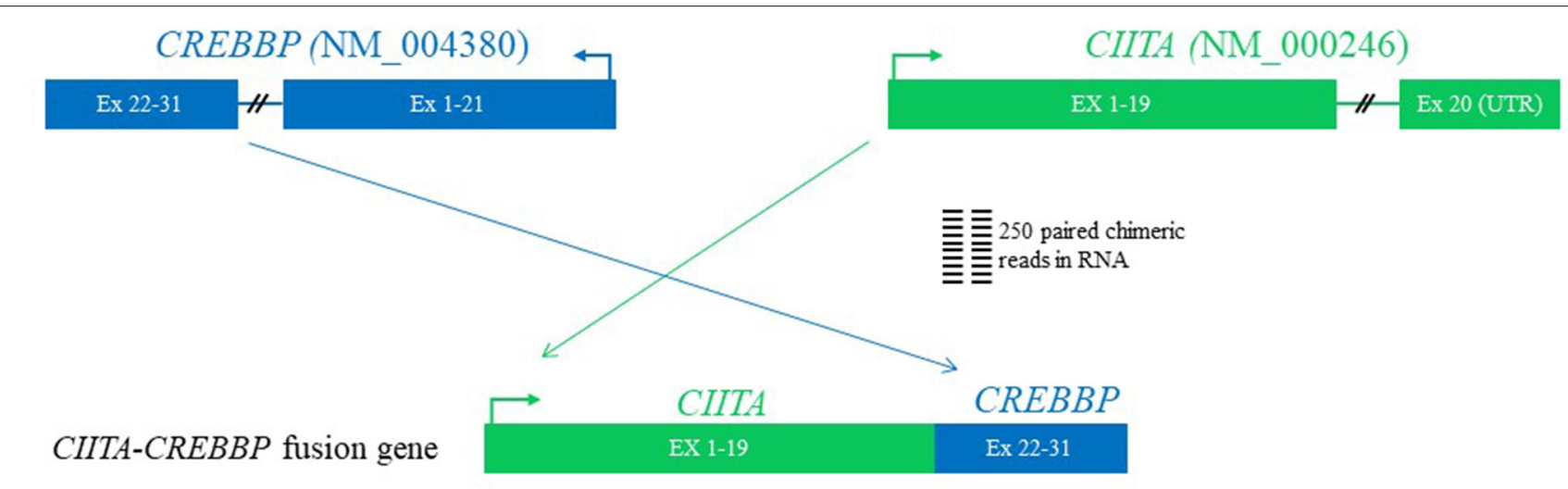

FIGURE 2 | The diagram of CIITA-CREBBP fusion by next generation sequencing using formalin-fixed paraffin-embedded tissue. The first 19 exons of CIITA are fused with exons of 22-31 of CREBBP. Since exon 19 of CIITA contains a stop codon and a portion of $3^{\prime}$-untranslated region (UTR) at 5' of exon 22 of CREBBP on the RNA, the fusion is postulated to generate a functional CIITA but a non-functional CREBBP.

\section{DISCUSSION}

Comprehensive genomic profiling using NGS revealed both expected and novel chromosomal and genomic abnormalities as aforementioned above. While presence of IgH-BCL2 fusion, CREBBP and STAT6 mutations are commonly seen in FL, CIITA-CREBBP has not yet been described in FL or in any lymphoma from the English literature so far, thus this is a novel finding. CREBBP and CIITA are both located at the same primary band on the short arm of chromosome 16, namely $16 \mathrm{p} 13.3$ and $16 \mathrm{p} 13.13$, respectively, thus the novel CIITA-CREBBP translocation discovered here was an intrachromosomal translocation via homologous recombination through the following two mechanisms: joining of CIITA and CREBBP with resultant deletion of the secondary band 3 through 13 within the portion of primary band 13; alternatively, the homologous recombination of CIITA and CREBBP was according to the Robersonian model. Given the fact that cells carrying this translocation were lymphoma cells, the chromosome involved was 16 , but not the commonly five acrocentric chromosome pairs, the first possibility that resulted in an unbalanced gene dosage are favored. The breakpoints of CIITA-CREBBP in the current case are distinct from the previously documented CIITA-BX648577 (10) in that the first 19 exons of CIITA are fused with exons 22-31 of CREBBP based on RNA read support (Figure 2). Although we do not have direct evidence to prove CIITA is functional, based on the genomic structure [exon 19 is the final coding exon of CIITA including the stop codon and a portion of the $3^{\prime}$ untranslated region (UTR)], and presence of HLA-DR protein expression as revealed by immunohistochemistry [a surrogate marker for CIITA) among the lymphoma cells (Figure 1I)], we conclude that the CIITACREBBP does not affect CIITA's function. However, CREBBP in the CIITA-CREBBP fusion most likely lost its expression and function (since the RNA transcript has a stop codon and portion of the 3'-UTR at the 5' of CREBBP exon 22), thus the lymphoma cells may have no CREBBP expression at all since we also observed a somatic mutation of CREBBP R1446H as well. Since
BCL2-IGH occurs very early in the development of FL during the $\mathrm{V}(\mathrm{D}) \mathrm{J}$ recombination (12), complete loss of function of CREBBP due to combined CIITA-CREBBP fusion and mutation is most likely an early but secondary event as well-demonstrated by Horton et al. (13). CREBBP mutations occur in $\sim 64 \%$ of FL, and were proposed to serve as early driver mutations $(13,14)$. Although it is a well-known phenomenon that radiation alone or in combination with chemotherapy increases the likelihood of secondary translocations and/or mutations (15), based on the early studies in particular $C R E B B P$ mutations were frequently detected in in situ follicular neoplasm $(13,14)$, the CREBBP mutation and translocation (CIITA-CREBBP) were most likely de novo rather than therapy-induced secondary events because of the additional facts that the patient had never received radiation therapy, and this biopsy were performed only after the first cycle of R-CHOP chemotherapy, too soon interval to evoke such genetic event.

The presence of CIITA-CREBBP in this particular FL is worthy notice not only because of its novelty, but also because this FL, while morphologically low-grade, exhibits higher ( $>30 \%$ ) proliferation index as demonstrated by Ki-67, thus this FL belongs to the so-called "low histologic grade FL with high proliferation index," which has been reported to be associated with clinical behavior more akin to grade $3 \mathrm{FL}$ according to the study by Wang et al. (16). More studies on the incidence of CIITA-CREBBP fusion are needed in order to assess the true relationship between the CIITA-CREBBP and low histologic grade FL with high proliferation index.

The other chromosomal translocation detected in this case, namely TBL1XR1-TP63, is reported in 5\% (6/115) and 1.2\% $(1 / 81)$ of DLBCL and FL, respectively (17), thus this is the only second documented FL with TBL1XR1-TP63. The breakpoints of TBL1XP1 (exons 1-7) and TP63 (exons 4-14) we observe here are similar to those reported from at least one case of DLBCLs but different from the reported FL by Scott et al. (17). Of interest, TBL1XP1-TP63 is also observed rarely in peripheral T-cell lymphoma [1.1\% (2/190)], where TP63 rearrangements rendered significant inferior overall survival (18). 
Besides the aforementioned three fusions, this patient also harbors multiple additional mutations of several genes including BCL2 and STAT6. BCL2 mutations occur in more than $50 \%$ of the FL. BCL2 mutations including A60V as seen in this patient result in overexpression of BCL2 and undoubtedly contribute to the lymphoma growth. Unlike BCL2 G101V, which confers resistance to BCL2 inhibitor in chronic lymphocytic leukemia (19), BCL2 A60V appears to have no adverse effect in overall survival and progression-free-survival on FL treated with rituximab (20). STAT6 mutations are found in $11 \%$ of FL and are implicated as a driver of FL pathogenesis (21).

\section{DATA AVAILABILITY STATEMENT}

The original contributions presented in the study are included in the article/supplementary material, further inquiries can be directed to the corresponding author/s.

\section{REFERENCES}

1. Rossi B, Noel P, Bruschi CV. Different aneuploidies arise from the same bridge-induced chromosomal translocation event in Saccharomyces cerevisiae. Genetics. (2010) 186:775-90. doi: 10.1534/genetics.110.120683

2. Nikitin D, Tosato V, Zavec AB, Bruschi CV. Cellular and molecular effects of non-reciprocal chromosome translocations in Saccharomyces cerevisiae. Proc Natl Acad Sci USA. (2008) 105:9703-8. doi: 10.1073/pnas.0800464105

3. Downing JR. The AML-ETO chimeric transcription factor in acute myeloid leukemia: biology and clinical significance. Br J Haematol. (1999) 106:296308. doi: 10.1046/j.1365-2141.1999.01377.x

4. Tosato V, West N, Zrimec J, Nikitin DV, Del Sal G, Marano R, et al. Bridgeinduced translocation between NUP145 and TOP2 yeast genes modles the genetic fusion between the human orthologs associated with acute myeloid leukemia. Front Oncol. (2017) 7:231. doi: 10.3389/fonc.2017.00231

5. Horsman DE, Gascoyne RD, Coupland RW, Coldman AJ, Adomat SA. Comparison of cytogenetic analysis, southern analysis and polymerase chain reaction for the detection of $\mathrm{t}(14 ; 18)$ in follicular lymphoma. Am J Clin Pathol. (1995) 103:472-8. doi: 10.1093/ajcp/103.4.472

6. Pelicci PG, Knowles DM, Magrath I, Dalla-Favera R. Chromosomal breakpoints and structural alterations of the c-myc locus differ in endemic and sporadic forms of Burkitt lymphoma. Prod Natl Acad Sci USA. (1986) 83:2984-8. doi: 10.1073/pnas.83.9.2984

7. Nagarajan UM, Bushey A, Boss JM. Modulations of gene expression by the MHC class II transactivator. J Immunol. (2002) 169:507888. doi: 10.4049/jimmunol.169.9.5078

8. Muhlethaler-Mottet A, Otten LA, Steimle V, Mach B. Expression of MHC class II molecules in different cellular and functional compartments is controlled by differential usage of multiple promotersof the transactivator CIITA. EMBO J. (1997) 16:2851-60. doi: 10.1093/emboj/16.10.2851

9. Ou Q, Lin L, Huang L, Chen F, Wu K, Lu P, et al. Persistence of MHC DR non-expression on swine cells by introduction of a mutated MHC class II transactivator gene: a comparison with the effect induced by antisense RNA. J Clin Immunol. (2004) 24:97-106. doi: 10.1023/B:JOCI.0000018068. 37899.6d

10. Steidl C, Shah SP, Woolcock BW, Rui L, Kawahara M, Farinha P, et al. MHC class II transactivator CIITA is a recurrent gene fusion partner in lymphoid cancers. Nature. (2011) 471:377-81. doi: 10.1038/nature09754

11. Vizmanos JL, Larrayoz MJ, Lahortiga I, Floristán F, Alvarez C, Odero MD, et al. $\mathrm{t}(10 ; 16)(\mathrm{p} 22 ; \mathrm{p} 13)$ and MORF-CREBBP fusion is a recurrent event in acute myeloid leukemia. Genes Chromosomes Cancer. (2003) 36:4025. doi: $10.1002 /$ gcc. 10174

12. Willis TG, Dyer MJ. The role of immunoglobulin translocations in the pathogenesis of B-cell malignancies. Blood. (2000) 96:808-22. doi: 10.1182/blood.V96.3.808

\section{ETHICS STATEMENT}

Ethical review and approval was not required for the study on human participants in accordance with the local legislation and institutional requirements. Written informed consent for participation was not required for this study in accordance with the national legislation and the institutional requirements.

\section{AUTHOR CONTRIBUTIONS}

HYW wrote the draft, took microphotographs, made Figure 1, and finalized the revision. ESS performed NGS and made Figure 2. AMG and CMM took care of the patient. ALF performed HLA-DR immunohistochemistry. All authors contributed to the article and approved the submitted version.

13. Horton SJ, Giotopoulos G, Yun H, Vohra S, Sheppard O, Bashford-Rogers R, et al. Early loss of Crebbp confers malignant stem cell properties on lymphoid progenitors. Nat Cell Biol. (2017) 19:1093-104. doi: 10.1038/ncb3597

14. Schmidt J, Ramis-Zaldivar JE, Bonzheim I, Steinhilber J, Müller I, Haake A, et al. CREBBP gene mutations are frequently detected in in situ follicular neoplasia. Blood. (2018) 132:2687-90. doi: 10.1182/blood-2018-03-837039

15. Barrios L, Caballín MR, Miró R, Fuster C, Guedea F, Subias A, et al. Cytogenetic effects of radiotherapy: frequency and types of chromosome aberrations. Int J Radiot Oncol Biol Phys. (1990) 19:371-5. doi: 10.1016/0360-3016(90)90546-V

16. Wang SA, Wang L, Hochberg EP, Muzikansky A, Harris NL, Hasserjian RP. Low histologic grade follicular lymphoma with high proliferation index: morphologic and clinical features. Am J Surg Pathol. (2005) 29:14906. doi: 10.1097/01.pas.0000172191.87176.3b

17. Scott DW, Mungall KL, Ben-Neriah S, Rogic S, Morin RD, Slack GW, et al. TBL1XR1-TP63: a novel recurrent gene fusion in B-cell non-Hodgkin lymphoma. Blood. (2012) 119:4949-52. doi: 10.1182/blood-2012-02-414441

18. Vasmatzis G, Johnson SH, Knudson RA, Ketterling RP, Braggio E, Fonseca R, et al. Genome-wide analysis reveals recurrent structural abnormalities of TP63 and other p53-related genes in peripheral T-cell lymphomas. Blood. (2012) 120:2280-9. doi: 10.1182/blood-2012-03-419937

19. Thangavadivel S, Byrd JC. Gly101Val BCL2 mutation: one step closer to understanding Venetoclax resistance in CLL. Cancer Discov. (2019) 3:3202. doi: 10.1158/2159-8290.CD-19-0029

20. Heut S, Szafer-Glusman E, Tesson B, Xerri L, Fairbrother WJ, Mukhyala $\mathrm{K}$, et al. BCL2 mutations do not confer adverse prognosis in follicular lymphoma patients treated with rituximab. Am J Hematol. (2017) 92:5159. doi: 10.1002/ajh.24701

21. Yildiz M, Li H, Bernard D, Amin NA, Ouillette P, Jones S, et al. Activating STAT6 mutations in follicular lymphoma. Blood. (2015) 125:66879. doi: 10.1182/blood-2014-06-582650

Conflict of Interest: AG is a consultant for Seattle Genetics and EUSA Pharma.

The remaining authors declare that the research was conducted in the absence of any commercial or financial relationships that could be construed as a potential conflict of interest.

Copyright (c) 2021 Wang, Sokol, Goodman, Feldman and Mulroney. This is an open-access article distributed under the terms of the Creative Commons Attribution License (CC BY). The use, distribution or reproduction in other forums is permitted, provided the original author(s) and the copyright owner(s) are credited and that the original publication in this journal is cited, in accordance with accepted academic practice. No use, distribution or reproduction is permitted which does not comply with these terms. 